\title{
Advancing Water Modelling through Networks and Engagement
}

\author{
Paul Lawrence and Jenny Riches \\ ${ }^{a}$ Queensland Department of Science, Information Technology and Innovation, Science Division \\ Email: paul.lawrence@dsiti.qld.gov.au
}

\begin{abstract}
The Queensland Water Modelling Network (QWMN) is an innovative approach to improve model capability, the integration of water resource, groundwater and water quality domains, the transferability and access to modelling tools, and to build modelling capacity and skills within Queensland. Following several independent reviews that highlighted gaps in modelling performance and planning, the Queensland Government embarked on a 4-year program to improve the strategic positioning of, and integration between, the discipline-based groups developing and applying modelling tools supporting policy and planning. These groups included water resource planning and flood management studies, groundwater resource assessments, and water quality modelling at paddock and catchment scales in the Great Barrier Reef, Murray-Darling Basin and Southeast Queensland. With an initial focus on models used within government, there was a strong desire to improve computational efficiencies, the $R \& D$ of models to more accurately reflect land and water transport processes, and embrace the use of visualisation to enhance model calibration, data interpretation and communication of modelling products. Access to calibrated models and their datasets, as well as best practice guidelines on modelling principles, and strengthening the governance arrangements of current modelling tools, were also identified as desirable outcomes through consultation across government agencies. While there is recognition of the role of models to inform policy, target-setting and planning initiatives within the Queensland Government, there is also the opportunity to capitalise on the strengths and capability in water modelling external to government within the research organisations, universities and the private sector. For this reason, the QWMN is a network in name and in nature, driving towards a 'community of practice' in modelling principles, the coordination of model R\&D efforts, synergies in model applications, and the establishment of access hubs through collaborative partnerships and engagements. For example, joint arrangements with universities are designed to embed students with government modelling practitioners working on real-world applications to support the water sector, while a catalogue of water models used within government is intended to enhance awareness of these tools and attract participation towards improving these models. This paper describes the thematic and governance arrangements underpinning the QWMN, the approaches to grow a network focusing on the water modelling and uptake, and capacity building through progressive collaborations, fellowships and co-investments. Achievements within its first year of operation and current investment portfolio are summarised. With the transition to Stage 2 in 2018, the QWMN also provides a catalyst for broader cross-institutional participatory processes, with the potential to be positioned as a national training and education collective in modelling research, development and applications.
\end{abstract}

Keywords: Model research and development, model arrangements, Source model, reef modelling, Queensland government 


\section{INTRODUCTION}

The well-known adage attributed to the British statistician George Edward Box (1979) that "All models are wrong, but some are useful" is a reminder about the separation between reality and the iterations between theory and practice. Box warns that excessive elaboration cannot obtain a 'correct' fit and that 'the scientist must be alert to what is importantly wrong. It is inappropriate to be concerned about mice when there are tigers aboard." (Box, 1976).

While this original observation was in reference to statistical models, the same sage advice and cautious approach remains relevant to water and catchment simulation models. Such words may not engender a high level of confidence in the minds of those who depend on the information derived from models to provide an evidence-base to assist policy makers and resource planners. Despite these known imperfections and uncertainties, the use and dependencies on modelling tools to inform decision making appears to be on the rise.

The reasons for this growth in model applications are numerous and varied (Kelly et al. 2013; Merritt et al. 2015), and include the following drivers:

- the information generated by the model may be at a greater spatial and temporal resolution than the available monitoring networks;

- many complex questions or 'wicked' problems cannot be addressed singularly by a monitoring program;

- models provide an ability to conduct structured scenarios or 'what if' analyses that enable policy options to be tested against environmental, social, economic and political considerations.

This is not to suggest that an unabated use of models is the solution to future resource planning, when indeed monitoring and modelling need to co-exist in a balanced, partnership arrangement to ensure uncertainties around information are minimised and the value of the information to assist decision making is maximised. All of which supports the second part of Box's reflection that '..some are useful'.

Queensland's natural resource and environmental planning activities have shown a long history in developing and applying hydrological and water quality simulation models. Current examples such as the Reef 2050 Water Quality Improvement Plan, the Water Resource Plans and the Brisbane Flood Management Strategy demonstrate both the reliance on models as tools for decision making, and the ability to develop frameworks for the operational deployment of models for resource planning linked to legislation, policy development and review.

Building on this recognised application of models, the purpose of this paper is to describe the next era in water resource modelling in Queensland with the establishment of the Queensland Water Modelling Network (QWMN). The network is an initiative of the Queensland government to modernise the research and development (R\&D) of water models and enhance the approaches, thematic frameworks and governance arrangements of its modelling environment. By its nature, the 'network' is designed to strengthen the linkages between model developers, practitioners and consumers rather than focusing on the infrastructure to create a 'modelling centre'. The intended approach is to grow a modelling network focusing on the integration, improvement, management, and uptake of models, and build capacity through progressive collaborations with other research providers, support fellowships and undertake joint activities of work through co-investments. Using summarised case studies from the portfolio of projects, this paper illustrates the achievements within its first year of operation, while forecast events and forums point to the opportunities to strategically position modelling in Queensland and grow a 'community of practice'.

\section{CONTEXT ON WATER MODELLING IN QUEENSLAND}

\subsection{The Greenfield Review}

In mid-2014, the Queensland Chief Scientist requested a review of water-related science and research within the Queensland Government, principally focusing on the Departments of Natural Resources and Mines (DNRM), and Science, Information Technology and Innovation (DSITI) but also including the Departments of Agriculture, Fisheries and Forestry (DAFF), Energy and Water Supply (DEWS) and Environment and Heritage Protection (DEHP) and Queensland Health $(\mathrm{QH})$. Some of the water related models used for natural resource, environmental and other applications, across various spatial scales of applications, are shown in Figure 1. 
The review identified several deficiencies within the current investment, particularly in the area of insufficient resources for R\&D modelling and strategic positioning to address future requirements by governments (Greenfield 2014). Following the review, two workshops were undertaken with key policy and planning agencies in Queensland to develop a collective and effective response to address these shortcomings. The workshops highlighted both the potential for a modelling network to be established as well as the challenges.

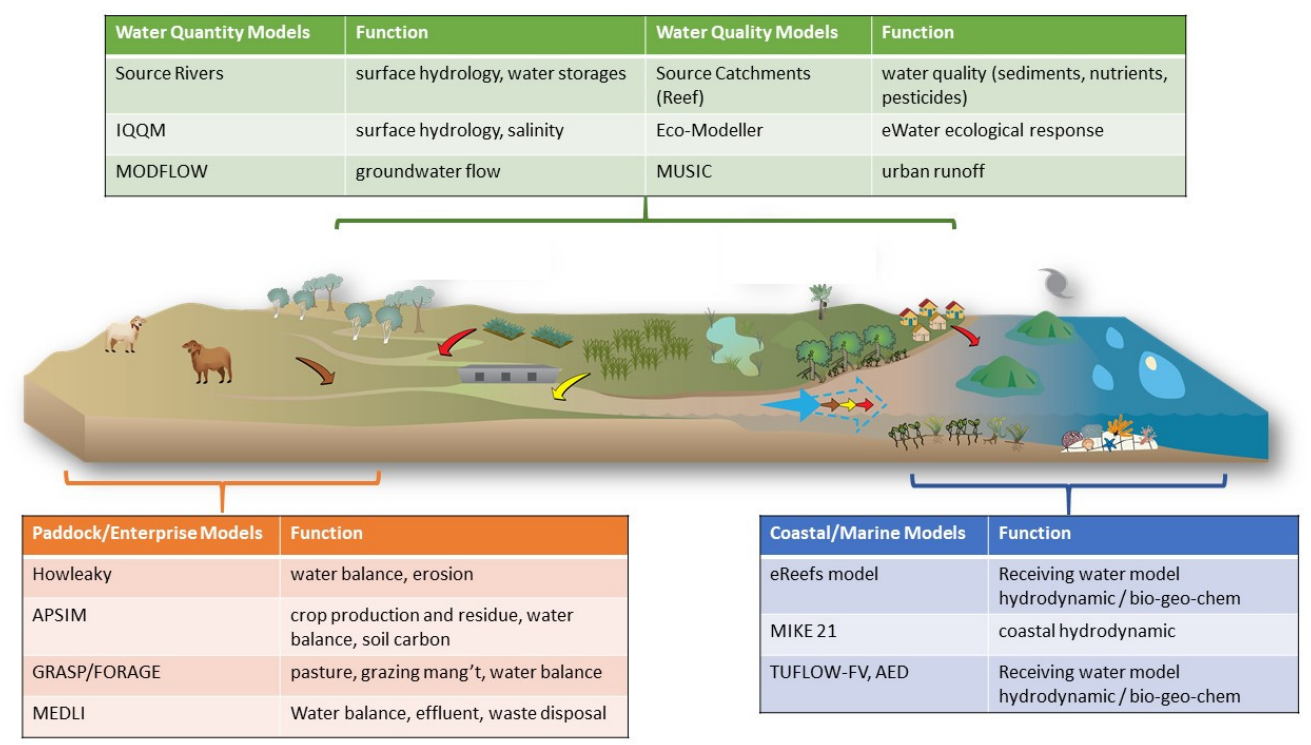

Figure 1. Some key water related models and functional applications within Queensland.

The review identified several deficiencies within the current investment, particularly in the area of insufficient resources for modelling R\&D and in strategic positioning to address future requirements by Government (Greenfield 2014). Following the review, two workshops were held with key policy and planning agencies in Queensland to develop a collective and effective response to address these shortcomings. The workshops highlighted both the potential for a modelling network to be established as well as the challenges facing it. The workshops also reinforced the view that, for the network to impact policy and management actions, it needed to integrate modelling, monitoring, and visualisations and interpretations, and where appropriate, seek to undertake a more consistent approach in the use of water models. Within government, there are numerous groups who either generate or utilise water-system models and associated data in decision-making, but these groups tend to be small, have quite different expertise and interests and exist in disciplinary silos (Greenfield, 2014). For example, water resource modelling, irrigation models, catchment flow models, flood mapping, groundwater models, salinity modelling, water quality and receiving water models may occur without necessarily looking for common linkages in data or modelling practices.

While identifying short term investment priorities was relatively straightforward across a range of modelling groups, the likelihood of achieving the identified additional goals and sustaining an active network, was low. There was neither a critical mass of individuals within government in any one area of water-system modelling, nor a sufficiently strong driver across the different sectors to provide confidence that, with the other pressures on agency personnel, ongoing network activity would occur. Many of the current funding programs using water modelling information are not aligned, are somewhat fragmented, and are almost entirely devoted to operational outcomes, with limited time and resources available to improve the modelling tools.

These observations lead to a key concept embedded in the recommended arrangements of the QWMN, namely that the Network move in a staged process from being internally focused to an externally focused network over four years, with an ultimate goal of establishing itself as the dominant national water-modelling network. In Stage 1 , the focus is on initiating priority activities to address key issues raised by relevant agencies and scientific modellers. For efficiency, it was also recommended that the Network adopt a 'light' but proactive level of governance utilising a small advisory group and technical experts, comprising representatives of agencies together with one or two external individuals. The current portfolio of start-up activities in Stage 1 based on discussions with different agencies is described later in the paper. 


\subsection{Paddock to Reef Modelling Reviews}

In 2015, the Queensland government through DNRM undertook an independent review of the paddock and catchment scale modelling being conducted to support the Reef Water Quality Protection Plan. In brief, the objectives of the review were to ensure that the modelling approach was adequate to meet the requirements for the Paddock-to-Reef (P2R) Program and annual Reef Report Card. In addition, the review aimed to ensure the program had sufficient rigor to represent paddock and catchment processes, and had sufficient support and capability to address identified gaps and improvements. While the review panel applauded the P2R approach for the depth of operational modelling to inform policy and government investments, one of the key observations from the review was the need to strengthen the linkages between paddock scale monitoring and modelling to enhance calibration and reduce uncertainties. It was also recommended that model computation efficiencies needed to be further explored, and that model management efficiencies (eg. data preparation, ingestion, generation and management) would yield valuable outcomes for a program that is regarded as a national leader in water quality modelling.

\section{QUEENSLAND WATER MODELLING NETWORK}

\subsection{Focus of the Modelling Network}

The objectives of the Queensland Water Modelling Network (QWMN) are to innovate the research and development capability of water models and enhance the integration of models to ensure Queensland is strategically positioned to maximise the value of these tools for planning and decision making. Project activities undertaken by the QWMN are designed to:

- improve the integration of modelling water processes for hydrology, groundwater and water quality;

- be state-wide, and link cross-scale modelling tools (paddock-enterprise-catchment-basin-regionalcoastal);

- $\quad$ support the integration of monitoring and modelling, particularly in priority catchments and basins;

- focus on modelling R\&D and innovation to improve the efficiency and effectiveness of operational modelling activities; and

- enhance co-investment and co-production with other modelling providers.

By focusing on the R\&D components of water models, the QWMN seeks to support the operational programs of modelling being undertaken by government programs such as the Reef Plan, Water Resource Planning, and SEQ water modelling, with improvements in model efficiency, model capability, visualisations and consistency benefitting those programs. The QWMN is funded for four years with an overall budget of $\$ 4$ million.

\subsection{Thematic Structure}

For Phase 1, five themes were identified:

- Model Integration - enhance the integration across surface water, groundwater and water quality, and across spatial scales (paddock, catchment and basin).

- Model Improvement - improve the R\&D and capability of models by incorporating known process understanding of water, sediment and nutrient movement.

- Model Management - develop and implement best practice principles of modelling, improve the mechanisms for data and model hosting, access and sharing, and maintain a catalogue of the scope, functionality, custodianship and applications of water models in use by government.

- Building Model Capacity and Uptake - implement programs to accelerate modelling skills and capability, and develop tools to improve data synthesis, communication, and visualisation.

- Communications and Engagement - implement strategies to engage universities, research organisations and private providers in the QWMN, develop co-investment opportunities and cross sector initiatives.

The context of the QWMN to act as a catalyst for continuous improvement in relation to the current modelling programs operating with the Queensland government is shown in Figure 2. 


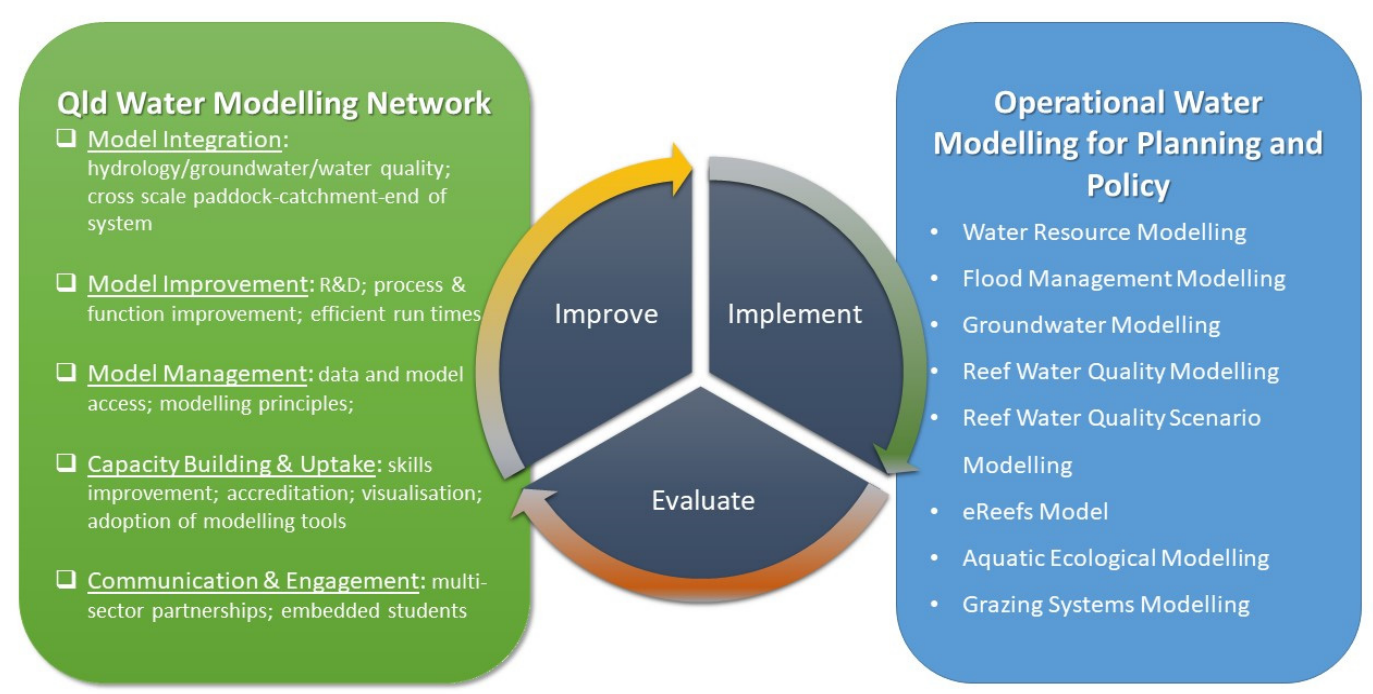

Figure 2. The QWMN with its five themes provides a mechanism for continuous improvement to support the operational water modelling programs in Queensland.

\subsection{Governance Arrangements}

As recommended by the Greenfield Report, the QWMN has adopted a light governance structure within Stage 1 to focus on the foundational activities and deliver a return to key government agencies. The three primary entities of the Network comprise a Core Group, a Steering Panel and five discipline-based technical working groups. These are briefly described below, and their functional linkages shown in Figure 3.

- Core Group - responsible for the Network's overall strategic direction, high-level approval of activities of work, interoperability with other governing committees and cross-organisational agreements, oversight for the transitional movement to Stage 2 and program delivery.

- Steering Panel - responsible for overseeing the integration of activities, information and products developed by the QWMN, and guiding the directions of the QWMN. The Steering Panel comprises the Core Group members, the Chairs of the five Technical Working Groups, and a senior executive from a user representative agency.

- Technical Working Groups - each of the technical working groups (TWG) undertakes the role of design, development and oversight of the priority tasks identified within their respective themes, including negotiations with technical suppliers from universities, research organisations and private sector providers. Each TWG comprises between 5-7 members with disciplinary skills and drawn from relevant agencies.

The QWMN has also adopted a range of procurement approaches to secure and deliver the program of activities. These procurement models include:

- traditional market-based invitation of quotes from suitably skilled providers for contractual services

- using the DSITI Standing Offer Arrangements (SOA) of listed preferred suppliers for designated areas of service;

- collaborative research agreements with universities, research organisations and/or consultants;

- commissioned works, where these are of minor activities or identified as sole supplier based on demonstrated specialist skills or expertise; and

- procurement of services under the Advance Queensland Small Business Innovation Research ${ }^{1}$.

\footnotetext{
${ }^{1}$ Advance Queensland Small Business Innovation Research (SBIR) provides commercial opportunities to innovators while solving challenges for government - see $\underline{h t t p}$ ://advance.qld.gov.au/small-business/sbir.aspx.
} 


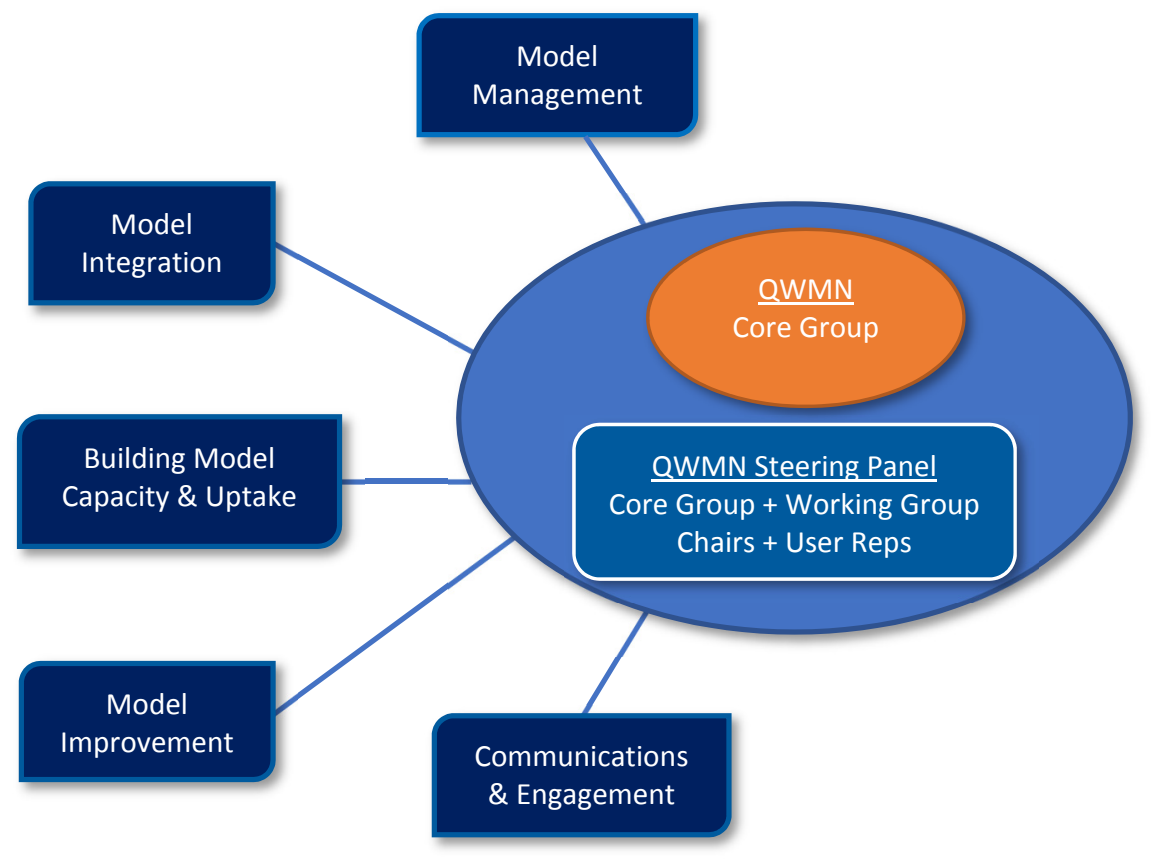

Figure 3. Governance arrangements for the Queensland Water Modelling Network

\section{CASE ACTIVITIES OF WORK}

The initial portfolio of priority R\&D modelling activities was developed by the TWGs in consultation with client agencies and recognising the recommendations from recent reviews of modelling programs. To date (August 2017), total project funding has been $\$ 1,050,000$, which has leveraged a co-investment of just over $\$ 500,000$, or approximately $50 \%$. For some projects, the leverage was greater than $100 \%$, reflecting the high priority and opportunity initiated through the Network for enhanced modelling outcomes. Three of the eight projects funded are multi-year.

Five case studies of the activities supported through the Network illustrate the enhancements and strategic investment being achieved through Stage 1 of the QWMN.

\subsection{Improvements to Dynamic SedNet}

The Dynamic SedNet model, which underpins Paddock to Reef catchment water quality modelling, is implemented as a series of plugins to the eWater Source model. While eWater Source had become a useful host for this modelling and leveraged rapid development efforts from elsewhere, the complexity of the Reef models resulted in slow run-times. Rapid run times were required to run many simulations for scenario assessment. It became increasingly apparent that Dynamic SedNet could be more efficiently run outside of the eWater Source graphical user interface. For example, a more computationally efficient approach could be implemented to run on Linux, as part of the National Computing Infrastructure (NCI) supercomputer. It could also be feasible to deploy a cloud-based version of the system as or when required. The work is being undertaken by a local supplier in close collaboration with modelling staff in DNRM and DSITI. The project also attracted co-funding from DSITI Queensland Hydrology and DNRM Natural Resources Division.

\subsection{Alterations to eWater core software coding}

This project supports improvements in the core eWater software to enhance model run time efficiency, performance and stability. The eWater Source modelling framework has been used as the tool to report on progress towards Great Barrier Reef Plan water quality targets and is becoming the preferred hydrological platform to inform water resource plans. The increasing complexity of the Source modelling framework has been severely impacting run time efficiency in parallel with a growing demand for more integrated model outputs. This project addresses several ongoing issues within the core eWater software which will generate benefits for the Paddock to Reef team, the Queensland Hydrology applications and the wider modelling community working in basin scale modelling. This project was co-funded by the QWMN and DNRM. 


\subsection{Enhanced data management and visualisation for Source Modelling}

Data visualisation capability is recognised as a fundamental component towards improving uptake, application and communication of model outputs and recommendations by policy makers and resource managers. This project focuses on the Great Barrier Reef and will develop a series of dashboard panels to provide rapid and standardised summary of model outputs for modellers and a range of external clients. The visualisation enhancements will include transport of water quality constituents from sub-catchment to catchment outlets and develop a process to upload subsets of model outputs to the web for rapid evaluation by modellers. Presentation on mobile devices will also be available. The work is being undertaken by a local supplier.

\subsection{Guidelines for best practice modelling principles}

The best practice modelling guide is designed as a reference document outlining current and a consistent approach to modelling principles for $R \& D$ to support the broader understanding and use of models in the government, private and university sectors. This includes coverage of the best practice methods for model calibration, validation, management, and mechanisms for data sharing. Prof. Tony Jakeman from ANU convened a facilitated workshop which brought together leaders in water modelling and data management to develop an integrated resource for use by modellers and non-modellers. The final report will be used as a key resource at workshops with external agencies and partners, as well as being shared on the QWMN website.

\subsection{Annotated catalogue of water models in use by the Queensland Government}

Consultation with key stakeholders identified a strong desire for a reference document describing the current portfolio of water models being used in government to support decision making by policy and planning agencies. This project has developed an annotated catalogue to serve as a reference document for new users and non-experts, and attracting broader interest and focus on improving the R\&D of models, particularly through providers external to government. The catalogue is being compiled by Griffith University, with an advanced version presented at the QWMN engagement workshop in August 2017.

\section{CONCLUDING OBSERVATIONS}

The QWMN represents a new era for water modelling in Queensland with a strong focus on improving the integration, R\&D and management of models. The Network is also building skills and capacity through the adoption of best modelling practice, attracting a greater focus on models used by government, and started the engagement with students as development opportunities for embedding with experienced practitioners to receive an accelerated experience in the fundamental understanding of models. By adopting a true network philosophy, the QWMN is growing the engagement of public, private and university sectors towards a community of practice in water modelling. With the transition to Stage 2, the QWMN also provides a catalyst for broader cross-institutional participatory processes, with the potential to be positioned as a national training and education collective in modelling research, development and applications.

\section{REFERENCES}

Box, G. E. P. (1976). Science and Statistics, Journal of the American Statistical Association, 71: 791799, DOI:10.1080/01621459.1976.10480949.

Box, G. E. P. (1979). Robustness in the strategy of scientific model building. in R.L. Launer and G.N. Wilkinson (eds.), Robustness in Statistics, Academic Press: pp.201-236.

Greenfield, P. (2014). Science Capability Audit - Water, Final Report, July 2014. Queensland Government Office of the Queensland Chief Scientist, Publications/Reviews and Audits: 36pp.

Kelly (Letcher), R.A., Jakeman, A.J., Barreteau, O. et al. (2013). 'Selecting among five common modelling approaches for integrated environmental assessment and management', Environmental Modelling and Software, vol. 47: pp. 159-181.

Merritt, W., Fu, B., Ticehurst, J. et al. (2015). 'Synthesising and evaluating the criteria of successful decision support models to support water resource assessment and management',21st International Congress on Modelling and Simulation (MODSIM2015), ed. Weber, T., McPhee, M.J. and Anderssen, R.S., The Modelling and Simulation Society of Australia and New Zealand Inc., Australia: p. 498. 